\title{
Validation in Swedish of Sydney Swallow Questionnaire
}

\author{
Beatriz Arenaz Búa $a^{1,2,3,4^{*}}$ and Margareta Bülow ${ }^{5,6,7}$
}

\begin{abstract}
Background: The aim of this study was to translate and adapt the Sydney Swallow Questionnaire to Swedish conditions and to evaluate the validity and test-retest reliability of the Swedish translation in patients with oropharyngeal dysphagia and in healthy controls.

Methods: The validation included 20 patients with swallowing problems and 20 controls matched in age and sex. Patients were assigned a Dysphagia Outcome and Severity Scale. Content, construct, discriminant and predictive validity and test-retest reliability were evaluated.

Results: The Swedish version of the Sydney Swallow Questionnaire was close to the original version, easy to fill in, and well accepted. The form fulfilled the criteria for content, construct, discriminant and predictive validity and test-retest reliability.

Conclusions: The Swedish translation of the Sydney Swallow Questionnaire proved to be a valid instrument to assess dysphagia symptoms and could be used in clinical settings.
\end{abstract}

Keywords: Oropharyngeal dysphagia, Validation, Questionnaire, Sydney Swallow Questionnaire

\section{Background}

Oropharyngeal dysphagia is common in an elderly population. It might be caused by morphological changes such as tumours or inflammation, secondary to neurological diseases or the result of aging. Video-Fluoroscopic Swallow Study (VFSS), videomanometry and flexible endoscopic evaluation of swallowing (FEES) reflect changes in the physiology and biomechanics of swallowing and are valuable tools in determining the extent of dysfunction, but do not take the patient's perspective into account.

Measurements of dysphagia severity are important when making management decisions and in the objective evaluation of treatment efficacy. Combining a self-report instrument with evaluation measures such as VFSS and FEES could contribute to these decisions.

Several questionnaires related to oropharyngeal dysphagia have been translated and validated from their original language (English) to other languages: Swallowing Quality

\footnotetext{
* Correspondence: barenazb@yahoo.com

'Division of Logopedics, Phoniatrics and Audiology, Jan Waldenströmsgata

18, SE- 20502 Malmö, Sweden

${ }^{2}$ Division of Ear, Nose and Throat Diseases, Head and Neck Surgery, Jan

Waldenströmsgata 18, SE- 20502 Malmö, Sweden

Full list of author information is available at the end of the article
}

of Life questionnaire (SWAL-QOL) [1-3] to French [4], Swedish [5], Chinese [6] and Dutch [7,8], Eating Assessment Tool (EAT-10) [9] to Spanish [10] and Italian [11], Dysphagia Handicap Index (DHI) [12] to Portuguese [13] and Arabic [14] and MD Anderson Dysphagia Inventory (MDADI) [15] to Italian [16] and Swedish [17]. EAT-10 has been validated in patients with a wide variety of causes of dysphagia, it is simple to complete and score. DHI is a 25 -item questionnaire, in which the patient can assign three responses for each question (never $=0$, sometimes $=2$, always $=4$ ) resulting in a score between 0 and 100. Moreover, patients rate their dysphagia assigning a score from 0 to 7 .

In Sweden there are currently two validated forms that address dysphagia symptoms: MDADI developed to assess dysphagia and quality of life in individuals with head and neck cancer and the SWAL-QOL that consists of 44 items and might be difficult for some patients to complete. We have some experience using the Self-report Symptom Inventory, known as Sydney Swallow Questionnaire (SSQ), see Additional file 1, and this is one of the reasons why we have chosen to validate it [18]. The questionnaire is well accepted, completed in a short time and less time consuming for the clinician in the everyday use, see 
Additional file 2. These are all important aspects to dysphagia patients and to clinicians with limited time.

When translating a form, a cross-cultural adaptation including the examination of cultural and linguistic differences is mandatory in order to obtain an equivalent instrument adapted to Swedish culture [19].

\section{Aim of the study}

- To translate and adapt SSQ to Swedish conditions.

- To evaluate the validity and test-retest reliability of the Swedish translation in patients with oropharyngeal dysphagia and in healthy controls.

\section{Methods \\ Inventory}

The SSQ is a self-report inventory with a maximum total score of 1700; a visual analogue scale appears immediately beneath all but one question (Q12). Each visual analogue scale is a horizontal, $100-\mathrm{mm}$ line anchored at each end by extreme statements representing normal function to the left and extreme dysfunction to the right (e.g., does not occur \& occurs all the time; no difficulty \& extreme difficulty). Participants were instructed to mark a single " $X$ " across the horizontal visual analogue scale at the point which they feel best represented the severity of the particular dysfunction, thus yielding a score of 0-100 for each, corresponding to a distance in millimetres from the origin of the visual analogue scale. In addition, one investigator delivered the written instructions verbally. No attempt was made to guide the patient as to where on the visual analogue scale he/she should make the mark.

The patients answered the SSQ on two occasions: In connection with visits to the Ear Nose and Throat (ENT) clinic or to the Radiology department and at home 3 weeks later. A stamped addressed envelope was given to the subjects for the return of SSQ to the contact person of the study [18].

Those responsible for the study have received formal approval for the translation and validation from the lead author of the SSQ. The translation has been performed by back- translation. The authors separately made a first translation from English to Swedish. In phase two the items with divergent translations were discussed until a consensus was reached. In phase three the SSQ was translated back to English by an independent, native English speaker, graduated in linguistics, who did not participate in the first and second phases. In phase four the SSQ was translated back into Swedish and a pilot group of four patients with swallowing disorders and four healthy subjects completed the questionnaire. In phase five, some of the formulations in the Swedish version of the questionnaire were altered according to the comments of the pilot group [19].

\section{Participants}

The final Swedish version was used, with approval by the ethical committee of the University of Lund (Dnr 2012/464), on 20 subjects without swallowing problems and on 20 patients with swallowing problems, both groups matched in age and sex. Information regarding the study was given to participants to obtain their written inform consent. All were older than 50 years and had adequate cognitive and language skills to comprehend study requirements. The SSQ has been validated in English in a cohort of head and neck patients [20]. None of the participants in our study had undergone previous head and neck surgery nor radiotherapy that might have influenced swallowing function. Controls were recruited when they visited the ENT department and completed the SSQ once.

Patients with oropharyngeal dysphagia for more than 3 months were included after the diagnose was confirmed with VFSS and a clinical evaluation by an otolaryngologist. After inclusion they were assigned a Dysphagia Outcome and Severity Scale (DOSS) score. This is a 7-point scale developed to systematically rate the severity of dysphagia based on VFSS and to make recommendations for diet level, independence level and type of nutrition. Level 7 is normal swallowing and level 1 stands for severe dysphagia [21].

Patients answered the SSQ twice. We included patients with neuromyogenic dysphagia and cricopharyngeal dysfunction (with and without Zenker's diverticulum), this last group was used to measure discriminant validity.

\section{Statistical methods}

All data were analyzed using SPSS 22 (c) Mac version. When a patient omitted more than 3 questions the inventory was excluded from further analysis, if 1 to 3 questions were not answered an estimated score for each omitted question was calculated based on the total score divided by the total possible score for the questions answered. Estimated scores for individual questions were only used for factor-analysis calculations, which requires a complete data set for each patient. We evaluated content, construct, discriminant and predictive validity and test-retest reliability. $\mathrm{P}$ values $<0.05$ (two-tailed) were regarded as significant [22-24].

\section{Content validity}

Content validity and internal consistency review whether the relative importance and choice of questions within the inventory are appropriate for the intended use of the SSQ. We chose factor analysis to examine the underlying relationships between the questions and to evaluate 
content validity. We have used the principal-components method with the orthotran/varimax rotation. We calculated the Kaiser-Meyer-Olkin as a measure of sampling adequacy and Bartlett's test of sphericity (if statistically significant it indicates that the relationships among the coefficients are not random). The factor analysis output presented a matrix of factor loadings. It is generally accepted that a factor loading greater than 0.3 is significant, but we selected 0.6 as cut-off for an individual question in the SSQ to be considered as part of a particular factor and it must not be represented in any other factor $[18,24]$. Factor analysis provides a communality summary that gives a measure of the variance of each question that can be accounted for the combination of all the factors, which overall should account at least for $75 \%$ of the total variance of the questionnaire. The total variance that each question contributes should be more than $0.6[18,24]$.

\section{Construct, discriminant and predictive validity}

Construct validity refers to whether an instrument measures the true clinical state of the patient. We hypothesized that the DOSS correlated with the SSQ and used Spearman's nonparametric correlations to confirm this.

Discriminant validity measures the SSQ ability to distinguish clinically significant differences in therapeutic responses over time, e.g. pre and postoperative scores.

We compared the SSQ score, using the Wilcoxon test, pre-operatively and 4 week post-operatively in 4 patients with Zenker's diverticulum treated with staples myotomy and 6 with cricopharyngeal dysfunction treated with balloon dilatation.

Predictive validity or known-groups validity refers in this case to whether SSQ can differentiate between patients with dysphagia and normal swallowers or patients with different severity of dysphagia. We have used the Mann Whitney $\mathrm{U}$ test to evaluate predictive validity.

\section{Test-retest reliability}

The test-retest reliability measures the ability of the SSQ to yield consistent scores over time, given that the clinical status of the patient remains stable. We evaluated the variability of the score within 3 weeks time using the Intraclass Correlation Coefficient (ICC) and limits of agreement (LOA), which is the 95\% confidence intervals of the mean of the individual differences between test and retest [22].

Ceiling and floor effects were assessed. A ceiling effect is said to occur when a high proportion of subjects in a study have maximum scores on the observed variable (the opposite is called floor effect). This makes discrimination among subjects on the top or the lower end of the scale impossible [25].

\section{Results}

\section{Descriptive statistics}

We recruited 20 controls and 20 patients with dysphagia, 10 men and 10 women, mean age 72 years in both groups. All responded the SSQ in less than $10 \mathrm{~min}$. One patient did not answer one question and one patient did not submit the postoperative questionnaire. None of the participants experienced difficulties in completing the questionnaire.

\section{Content validity (internal consistency)}

Kaiser-Meyer-Elkin was 0.75 indicating a sufficient sample size for the number of questions in the questionnaire. Bartlett's test of sphericity was significant $\mathrm{p}<0.001$.

The factor analysis matrix, showed that all questions except Q12 contributed significantly to factor 1 (Table 1). Question 12 (related to how long time does it take to eat) was the sole contributor to factor 3. All questions had a communality loading $>0.6$ and $85 \%$ of the variance in response is explained by the 4 major factors identified by the analysis, $61 \%$ for the first factor (dysphagia).

\section{Construct, discriminant and predictive validity}

Spearman correlations coefficient was $-0.70, \mathrm{p}<0.001$ confirming construct validity (Figure 1 ).

Regarding discriminant validity the preoperative mean value was 722, median 634 and postoperative mean 313, median 234 and the Wilcoxon signed ranks test was significant with $\mathrm{p}=0.002$ (Figure 2).

Predictive validity: as hypothesized, dysphagic patients scored significantly higher on SSQ, $\mathrm{p}<0.001$. The mean score for controls was 51 , median 48; minimum score was 5 and maximal 102. The mean score for patients was 638, median 607; minimum score was 113 and maximal 1489 (Figure 3).

\section{Test-retest reliability}

The ICC for patient scores within 3 weeks was 0.98 , 95\% CI (0.96-0.99) significant $\mathrm{p}<0.001$ (Table 2), 5 questions had ICC <0.7: Q1 0.63, Q3 0.64, Q8 0.53 and Q12 0.61.

\section{Discussion}

A self-report instrument is commonly used to assess patient reported outcome, it guarantees that questions are asked in a standardized manner, and facilitates comparisons within and between groups. These inventories are designed to measure either health-related quality of life (HRQoL) or functional health status (FHS), HRQoL refers to the perception individuals may have on their health taking into account social, functional and psychological issues, whereas FHS quantifies the symptomatic severity of a disease (in this case dysphagia) on particular functional aspects. DHI, MDADI and SWALQOL are HRQoL questionnaires, EAT-10 and SSQ are 
Table 1 Summary of the factor analysis matrix with communality summary in patients, $\mathbf{n}=20$

\begin{tabular}{llllll}
\hline Question & Factor $\mathbf{1}$ dysphagia & Factor $\mathbf{2}$ & Factor $\mathbf{3}$ & Factor $\mathbf{4}$ & Communality summary loading \\
\hline 1 & 0.788 & 0.388 & 0.239 & -0.106 & 0.840 \\
2 & 0.837 & -0.345 & -0.184 & -0.153 & 0.877 \\
3 & 0.809 & -0.362 & 0.066 & -0.312 & 0.887 \\
4 & 0.889 & -0.248 & 0.189 & -0.079 & 0.893 \\
5 & 0.805 & 0.176 & 0.424 & -0.249 & 0.922 \\
6 & 0.786 & 0.343 & 0.196 & -0.282 & 0.852 \\
7 & 0.622 & 0.455 & -0.498 & 0.170 & 0.871 \\
8 & 0.625 & 0.498 & 0.120 & 0.418 & 0.827 \\
9 & 0.765 & 0.124 & -0.003 & -0.258 & 0.667 \\
10 & 0.781 & 0.099 & 0.000 & 0.449 & 0.821 \\
11 & 0.858 & -0.293 & -0.225 & 0.243 & 0.932 \\
12 & 0.336 & -0.287 & 0.759 & 0.346 & 0.891 \\
13 & 0.812 & -0.452 & -0.036 & 0.251 & 0.928 \\
14 & 0.853 & 0.030 & -0.227 & 0.067 & 0.785 \\
15 & 0.822 & -0.322 & -0.310 & -0.012 & 0.876 \\
16 & 0.914 & 0.175 & 0.039 & 0.050 & 0.871 \\
17 & 0.804 & 0.140 & -0.166 & -0.230 & 0.747 \\
Variance & 61.2 & 9.5 & 8.3 & 6.2 & \\
\hline
\end{tabular}

FHS questionnaires. For optimal use of both types of inventories, it is necessary to combine psychometric and utility approaches [26-28].

The Swedish version of SSQ was well accepted, the response rate was high, and the number of missing items was very low (only Q6 in patient 19). The results indicated that the translation of SSQ was easy to manage and close to the original. It took less time to answer (less than 10 minutes) and score (less than

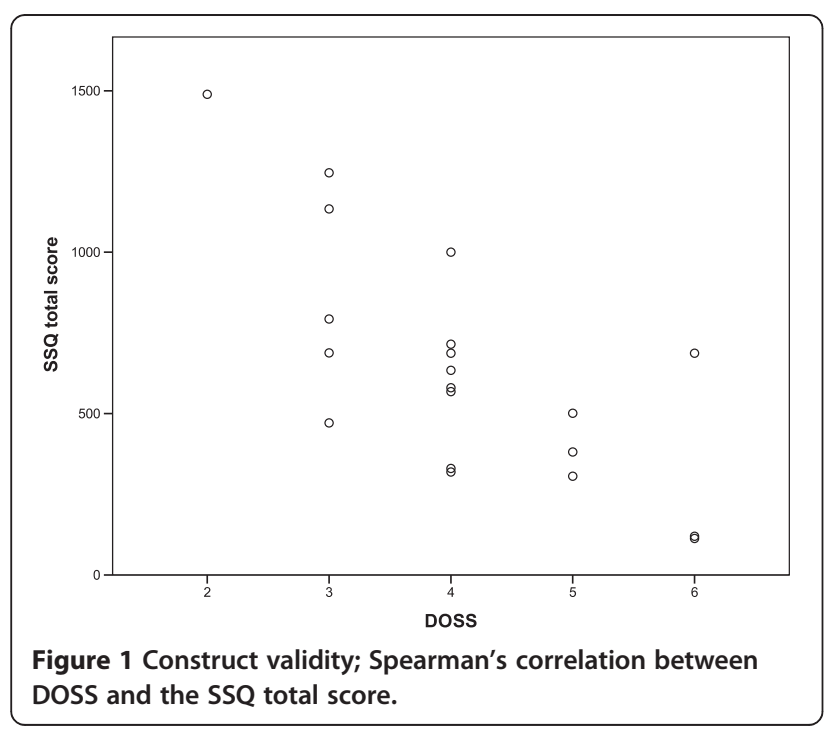

4 minutes), compared to SWAL-QOL and the MDADI. It had good test-retest reliability. The ICC for the total score was 0.98. All the questions reached the level 0.7 except Q1 (grade of dysphagia), Q3 (difficulty to swallow thick liquids), Q8 (difficulty to initiate the swallowing) and Q12 (how long does it take to eat), (Table 2).

Our sample was small which might be a limitation in our study, but the Kaiser-Meyer-Olkin was 0.75 , indicating

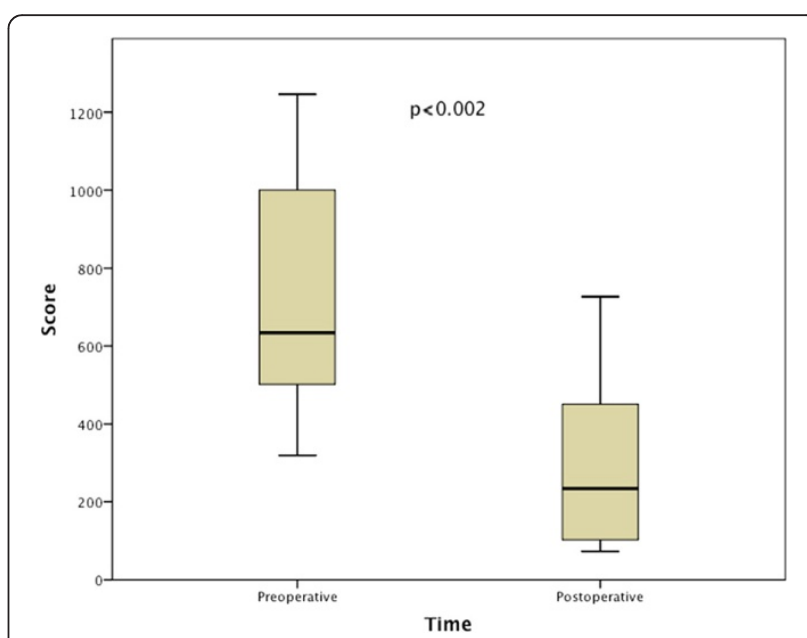

Figure 2 Discriminant validity; Wilcoxon signed rank test, showing pre-operative and 1 month post-operative comparison. 


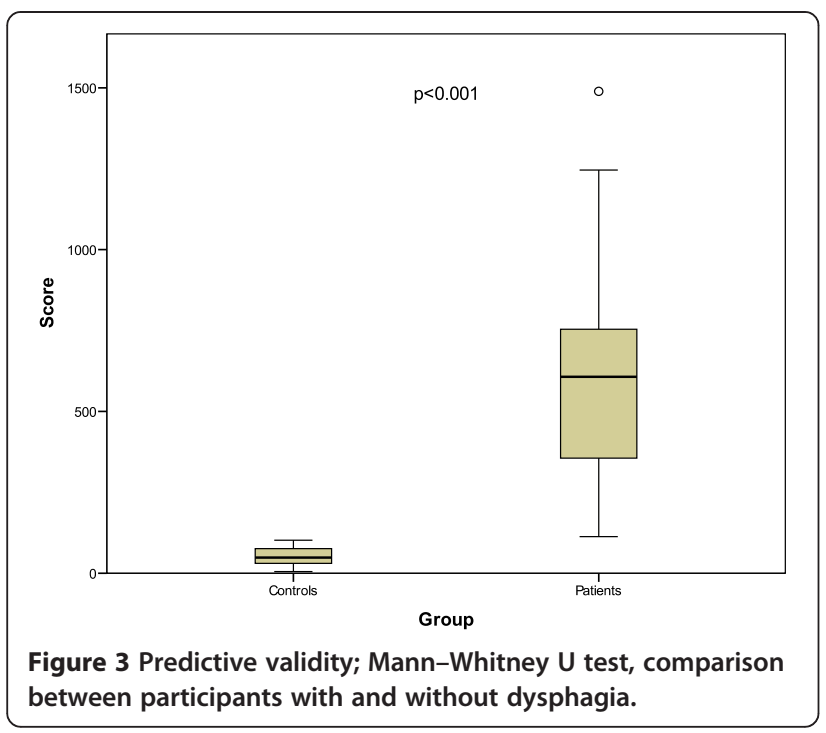

a sufficient sample for the number of questions in our questionnaire. The Swedish SSQ satisfied as well criteria for content, construct, discriminant, and predictive validity. Regarding content validity four factors accounted for $85 \%$ of its variance, the dominant factor (dysphagia) accounted for $61 \%$, slightly better than in the original article that was $59 \%$.

Table 2 Summary of the test-retest reliability, using the Intraclass Correlation Coefficient (ICC), confidence interval $(\mathrm{Cl})$

\begin{tabular}{llll}
\hline & ICC & $95 \%$ Cl & P-value \\
\hline Total score & 0.98 & $0.95-0.99$ & $<0.001$ \\
Q1 & 0.63 & $0.27-0.83$ & 0.001 \\
Q2 & 0.86 & $0.28-0.84$ & $<0.001$ \\
Q3 & 0.64 & $0.29-0.84$ & 0.001 \\
Q4 & 0.94 & $0.85-0.97$ & $<0.001$ \\
Q5 & 0.88 & $0.72-0.95$ & $<0.001$ \\
Q6 & 0.93 & $0.82-0.97$ & $<0.001$ \\
Q7 & 0.85 & $0.65-0.94$ & $<0.001$ \\
Q8 & 0.53 & $0.12-0.78$ & 0.007 \\
Q9 & 0.74 & $0.45-0.89$ & $<0.001$ \\
Q10 & 0.87 & $0.71-0.95$ & $<0.001$ \\
Q11 & 0.76 & $0.50-0.89$ & $<0.001$ \\
Q12 & $0.25-0.83$ & 0.002 \\
Q13 & 0.62 & $0.79-0.96$ & $<0.001$ \\
Q14 & 0.91 & $0.57-0.92$ & $<0.001$ \\
Q15 & 0.80 & $0.83-0.97$ & $<0.001$ \\
Q16 & 0.93 & $0.47-0.89$ & $<0.001$ \\
Q17 & 0.75 & $0.58-0.92$ & 0.001 \\
\hline
\end{tabular}

The total inventory score showed a -0.70 correlation with the DOSS, showing excellent construct validity. The correlation is negative: DOSS score decreases (level 1) and SSQ score increases (maximum 1700) when dysphagia severity rises. Wallace et al. calculated the construct validity correlated the SSQ with a global assessment score obtaining a positive linear correlation 0.69 [18].

Discriminant validity is important for using the inventory to measure responses to treatment and to establish the efficacy of that treatment. The mean preoperative total score decreased by an average of $60 \%$ postoperatively, $10 \%$ less than in the original article, but our study includes patients treated both with myotomy and balloon dilations and they probably differ in their postoperative results.

Subjects with dysphagia had significantly higher scores than the age- and gender-matched control group, suggesting very good predictive validity that helps to distinguish between individuals with/without dysphagia, which is central in a broader use. The cut-off score for dysphagia in our version of SSQ is 111, this is the controls mean total score plus two standard deviations $(51+(2 \times 30) \geq 111)$. Score values higher than 111 should be considered as pathological in our validation. However, in Wallace et al. SSQ the cut-off score is 193 for 19 controls with a mean age of 62 .

Floor and ceiling effects were not found in the Swedish SSQ. They were not reported in the SSQ original version.

By performing a Swedish version and validation of SSQ we have obtained a useful tool to record patient reported outcome of swallowing problems. This self-report instrument is not only easy for the patients to use, but also very efficient for the clinician.

\section{Conclusions}

The Swedish version of the SSQ seems to be a reliable and consistent instrument for the assessment of subjective dysphagia symptoms. The availability of validated patient reported outcome instruments such as the SSQ might be an important contribution to both research and screening of dysphagia in Sweden.

\section{Additional files}

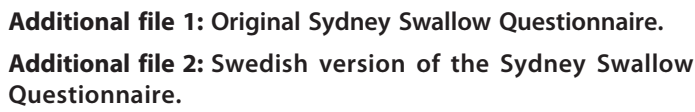

\section{Abbreviations}

Cl: Confidence intervals; DHI: Dysphagia Handicap Index; DOSS: Dysphagia Outcome and Severity Scale; EAT-10: Eating Assessment Tool; ENT: Ear Nose and Throat; FEES: Flexible Endoscopic Evaluation of Swallowing; FHS: Functional Health Status; HRQoL: Health-Related Quality of Life; ICC: Intraclass Correlation Coefficient; MDADI: MD Anderson Dysphagia Inventory; SWAL-QOL: Swallowing Quality of Life Questionnaire; SSQ: Sydney Swallow Questionnaire; VFSS: Video-Fluoroscopic Swallow Study; Q: Question. 


\section{Competing interests}

The authors declare that they have no competing interests.

\section{Authors' contributions}

$\mathrm{BA}$ and $\mathrm{MB}$ conceived of the study, participated in its design and coordination, collection of data and drafted the manuscript. BA performed the statistical analysis and interpretation of data. All authors have read and approved the final manuscript.

\section{Acknowledgements}

We thank Helene Jakobsson who provided statistical support and Professor Olle Ekberg for revising the manuscript critically.

\section{Author details}

${ }^{1}$ Division of Logopedics, Phoniatrics and Audiology, Jan Waldenströmsgata 18, SE- 20502 Malmö, Sweden. ${ }^{2}$ Division of Ear, Nose and Throat Diseases, Head and Neck Surgery, Jan Waldenströmsgata 18, SE- 20502 Malmö, Sweden. ${ }^{3}$ Department of Clinical Sciences, Lund University, Jan Waldenströmsgata 18, SE- 20502 Malmö, Sweden. ' Skane University Hospital, Jan Waldenströmsgata 18, SE- 20502 Malmö, Sweden. ${ }^{5}$ Diagnostic Centre of Imaging and Functional Medicine, SE- 20502 Malmö, Sweden. 'Department of Clinical Sciences, Lund University, SE- 20502 Malmö, Sweden. ${ }^{7}$ Skane University Hospital Malmö, SE- 20502 Malmö, Sweden.

Received: 23 July 2014 Accepted: 10 October 2014

Published: 21 October 2014

\section{References}

1. McHorney CA, Bricker DE, Kramer AE, Rosenbek JC, Robbins J, Chignell KA, Logemann JA, Clarke C: The SWAL-QOL outcomes tool for oropharyngeal dysphagia in adults: I. conceptual foundation and item development. Dysphagia 2000, 15(3):115-121.

2. McHorney CA, Bricker DE, Robbins J, Kramer AE, Rosenbek JC, Chignell KA: The SWAL-QOL outcomes tool for oropharyngeal dysphagia in adults: II. item reduction and preliminary scaling. Dysphagia 2000, 15(3):122-133.

3. McHorney CA, Robbins J, Lomax K, Rosenbek JC, Chignell K, Kramer AE, Bricker DE: The SWAL-QOL and SWAL-CARE outcomes tool for oropharyngeal dysphagia in adults: III. documentation of reliability and validity. Dysphagia 2002, 17(2):97-114.

4. Khaldoun E, Woisard V, Verin E: Validation in French of the SWAL-QOL scale in patients with oropharyngeal dysphagia. Gastroenterol Clin Biol 2009, 33(3):167-171.

5. Finizia C, Rudberg I, Bergqvist $H$, Ryden A: A cross-sectional validation study of the Swedish version of SWAL-QOL. Dysphagia 2012, 27(3):325-335.

6. Lam PMLC: The validation of the Chinese version of the Swallow Quality-of-Life Questionnaire (SWAL-QOL) using exploratory and confirmatory factor analysis. Dysphagia 2011, 26(2):117-124.

7. Bogaardt HC, Speyer R, Baijens LW, Fokkens WJ: Cross-cultural adaptation and validation of the Dutch version of SWAL-QoL. Dysphagia 2009, 24(1):66-70.

8. Lemmens JBG, Limburg M, Beurskens AJ: The feasibility and test-retest reliability of the Dutch Swal-Qol adapted interview version for dysphagic patients with communicative and/or cognitive problems. Qual Life Res 2013, 22(4):891-895.

9. Belafsky PC, Mouadeb DA, Rees CJ, Pryor JC, Postma GN, Allen J, Leonard RJ: Validity and reliability of the Eating Assessment Tool (EAT-10). Ann Otol Rhinol Laryngol 2008, 117(12):919-924.

10. Burgos R, Sarto B, Segurola H, Romagosa A, Puiggros C, Vazquez C, Cardenas G, Barcons N, Araujo K, Perez-Portabella C: Translation and validation of the Spanish version of the EAT-10 (Eating Assessment Tool-10) for the screening of dysphagia. Nutr Hosp 2012, 27(6):2048-2054.

11. Schindler A, Mozzanica F, Monzani A, Ceriani E, Atac M, Jukic-Peladic N, Venturini C, Orlandoni P: Reliability and validity of the Italian Eating Assessment Tool. Ann Otol Rhinol Laryngol 2013, 122(11):717-724.

12. Silbergleit AK, Schultz L, Jacobson BH, Beardsley $T$, Johnson AF: The Dysphagia handicap index: development and validation. Dysphagia 2012, 27(1):46-52

13. Goncalves MI, Remaili CB, Behlau M: Cross-cultural adaptation of the Brazilian version of the Eating Assessment Tool - EAT-10. CoDAS 2013, 25(6):601-604
14. Farahat M, Malki KH, Mesallam TA, Bukhari M, Alharethy S: Development of the Arabic Version of Dysphagia Handicap Index (DHI). Dysphagia 2014, 29:459-467.

15. Chen AY, Frankowski R, Bishop-Leone J, Hebert T, Leyk S, Lewin J, Goepfert $H$ : The development and validation of a dysphagia-specific quality-of-life questionnaire for patients with head and neck cancer: the M. D. Anderson dysphagia inventory. Arch Otolaryngol Head Neck Surg 2001, 127(7):870-876.

16. Schindler A, Borghi E, Tiddia C, Ginocchio D, Felisati G, Ottaviani F: Adaptation and validation of the Italian MD Anderson Dysphagia Inventory (MDADI). Rev Laryngol Otol Rhinol (Bord) 2008, 129(2):97-100.

17. Carlsson S, Ryden A, Rudberg I, Bove M, Bergquist H, Finizia C: Validation of the Swedish M. D. Anderson Dysphagia Inventory (MDADI) in patients with head and neck cancer and neurologic swallowing disturbances. Dysphagia 2012, 27(3):361-369.

18. Wallace $K L$, Middleton S, Cook IJ: Development and validation of a self-report symptom inventory to assess the severity of oral-pharyngeal dysphagia. Gastroenterology 2000, 118(4):678-687.

19. Guillemin F, Bombardier C, Beaton D: Cross-cultural adaptation of health-related quality of life measures: literature review and proposed guidelines. J Clin Epidemio/ 1993, 46(12):1417-1432.

20. Dwivedi RC, St Rose S, Roe JW, Khan AS, Pepper C, Nutting CM, Clarke PM, Kerawala CJ, Rhys-Evans PH, Harrington KJ, Kazi R: Validation of the Sydney Swallow Questionnaire (SSQ) in a cohort of head and neck cancer patients. Oral Oncol 2010, 46(4):e10-e14.

21. O'Neil KH, Purdy M, Falk J, Gallo L: The dysphagia outcome and severity scale. Dysphagia 1999, 14(3):139-145.

22. Carmines EGZR: Reliability and validity assessment. London: Sage; 1979:15-27.

23. Altman DG: Practical Statistics for Medical Research. London: Chapman \& Hall/CRC; 1999:179-223.

24. Pett MLN, Sullivan JJ: Making Sense of Factor Analysis: the Use of Factor Analysis for Instrument Development in Health Care Research. Thousand Oaks, California: Sage Publications, Inc; 2003:1-12. 77-83, 208-209, 226-240.

25. Po ALW: Dictionary of Evidence-based Medicine. Abingdon, Oxon: Radcliffe Publishing; 1998:20.

26. Speyer R, Cordier R, Kertscher B, Heijnen BJ: Psychometric properties of questionnaires on functional health status in oropharyngeal dysphagia: a systematic literature review. BioMed Res Int 2014, 2014:458678.

27. Timmerman AA, Speyer R, Heijnen BJ, Klijn-Zwijnenberg IR: Psychometric characteristics of health-related quality-of-life questionnaires in oropharyngeal dysphagia. Dysphagia 2014, 29(2):183-198.

28. Ferrans CE, Zerwic JJ, Wilbur JE, Larson JL: Conceptual model of healthrelated quality of life. J Nurs Scholarsh 2005, 37(4):336-342.

\section{doi:10.1186/1756-0500-7-742}

Cite this article as: Arenaz Búa and Bülow: Validation in Swedish of Sydney Swallow Questionnaire. BMC Research Notes 2014 7:742.

\section{Submit your next manuscript to BioMed Central and take full advantage of:}

- Convenient online submission

- Thorough peer review

- No space constraints or color figure charges

- Immediate publication on acceptance

- Inclusion in PubMed, CAS, Scopus and Google Scholar

- Research which is freely available for redistribution

Submit your manuscript at www.biomedcentral.com/submit
C Biomed Central 